\title{
One- and two-photon ionization of hydrogen atom embedded in Debye plasmas
}

\author{
T. N. Chang ${ }^{1}$, T. K. Fang ${ }^{2}$, and Y. K. Ho ${ }^{3 *}$ \\ ${ }^{1}$ Department of Physics and Astronomy, University of Southern California, \\ Los Angeles, CA 90089-0484, U.S.A. \\ ${ }^{2}$ Department of Physics, Fu Jen Catholic University, Taipei, Taiwan, 242, \\ $\mathrm{ROC}$ \\ ${ }^{3}$ Institute of Atomic and Molecular Sciences, Academia Sinica, P. O. Box \\ 23-166, Taipei, Taiwan, 106, ROC
}

\begin{abstract}
We present a detailed analysis of the plasma-induced resonance-like atomic structures near the ionization threshold in one- and two-photon ionization of hydrogen atom. Such resonance-like structures result from the migration of the upper bound excited states of bound-bound atomic transitions into the continuum due to the less attractive screened Coulomb potential which simulates the external environmental effect for an atom embedded in Debye plasma. The change from the resonance-like narrow structures into broad continuous spectra as the plasma effect increases could be accounted for by the overlap between the respective wavefunctions of the atomic electron in the initial state and its corresponding outgoing ionized state in the continuum.
\end{abstract}

PACS number(s); 52.25.Os, 32.80.Fb

*ykho@pub.iams.sinica.edu.tw 


\section{Introduction}

With the recent development in laser plasmas produced by laser fusion in laboratories [1], the continued interest of helium abundance in astrophysics plasmas [2], and the activities on cold plasmas [3], it is important to have accurate atomic data available in the literature for atoms/ions in various plasma environments. Considerable works [4-13] have appeared in the literatures for atomic processes in plasma environments due to the screening effect on the plasma-embedded atomic systems, using the Debye-Hückel model [14] with a screened Coulomb potential

$$
V_{s}(r ; D)=-\frac{Z e^{2}}{r} e^{-r / D}
$$

where the parameter $D$ is the Debye-Hückel length (or Debye length). The potential $V_{s}$ is derived from the screened potential in the original DebyeHückel approximation by taking the limit of zero radius for the Debye sphere $[15,16]$. The Debye length characterizes the screening of the nuclear charge experienced by the atomic electron due to the plasma in the outer region of an atom and is given in Bohr radius $a_{o}$ by (see, e.g., Eq. (2.5) of [15])

$$
D=1.304 \times 10^{8}(T / n)^{1 / 2} a_{o}
$$

or, alternatively, it can be expressed in the unit of $\mathrm{cm}$ by $D=6.90(T / n)^{1 / 2}$ where $T$ and $n$ are the plasma temperature (on Kelvin scale) and density (in $\mathrm{cm}^{-3}$ ), respectively.

The atomic excitation, ionization, and photoionization processes play a key role in the plasmas diagnostics. It was shown in the studies of inelastic and elastic collision processes in plasmas [17] that the cross sections are reduced by plasma screening effects in electron-ion interactions. In addition, Jung and coworkers have investigated the $1 \mathrm{~s}-2 \mathrm{~s}, 1 \mathrm{~s}-2 \mathrm{p}$ and $2 \mathrm{~s}-2 \mathrm{p}$ excitation cross sections for electron atom/ion scattering and reported various screening effects on such processes [17]. More recently, studies were carried out on atomic processes in Debye plasmas, including calculations of photoionization of atoms using the method of complex-coordinate rotation $[9,18]$, the cross sections of charge exchange and ionization in ion-atom collisions using the classical trajectory Monte Carlo (CTMC) approach [10], and two/threephoton bound-bound transitions for the hydrogen atom in plasmas [11]. 
The main purpose of this paper is to present the results of an investigation on one- and two-photon ionizations on plasma-embedded hydrogen atom. In particular, we will focus on the plasma-induced resonance-like atomic structures in the continuum due to the migration of the upper bound excited states into the continuum with less attractive and shorter-ranged potential as the Debye length decreases. Such plasma-induced spectrum first appears as a narrow resonance-like structure when the upper bound excited atomic state of a specific bound-bound transition (e.g., $1 s \rightarrow n p$ transition for hydrogen atom) first moves across the ionization threshold into the continuum due to the plasma effect. It gradually changes from a narrow resonance-like structure into a broad continuous spectrum as the Debye length decreases further. In fact, from our investigation, similar resonance-like structures are also expected in the two-photon ionization spectrum. We will present a detailed analysis to understand this general feature of plasma-induced resonance-like atomic structures by examining the variation of the one-electron wavefunction of the outgoing ionized electron and its spatial overlap with the wavefunction of the initial bound electron.

\section{Theory and Calculation Procedure}

The single photoionization cross section $\sigma$ from an initial state $i$ to a final continuum $f$ with an outgoing electron of momentum $k$, using the discretized B-spline based atomic orbits, is expressed in terms of the oscillator strength $f_{f i}$ by the expression $[19,20]$

$$
\sigma=\frac{4 \pi \alpha}{k A_{o}^{2}} f_{f i}
$$

where $A_{o}$ is the amplitude of the outgoing one-electron oscillating wavefunction and $\alpha$ is the fine structure constant. The standard per unit energy normalization constant $(2 / \pi k)^{1 / 2}$ for the outgoing electron is replaced by $A_{o}$ for the B-spline based atomic wavefunctions confined in a sphere of radius $R$. For the one-electron atoms, the one-particle radial wavefunction $\chi_{\nu \ell}(r)$ for a state $\nu \ell$ corresponding to orbital angular momentum $\ell$ and an energy

eigenvalue $\epsilon_{\nu \ell}$ is generated from

$$
h_{o}(r ; D) \chi_{\nu \ell}=\epsilon_{\nu \ell} \chi_{\nu \ell}
$$


where the one-electron Hamiltonian is given by

$$
h_{o}(r ; D)=\frac{p^{2}}{2 m}+V_{s}(r ; D),
$$

and the operator $p$ represents the momentum of the electron. For the outgoing ionized electron, $\nu$ represents the energy $\epsilon=k^{2}$ in $R y$ unit.

The oscillator strength $f_{f i}$ and its corresponding photoionization cross section are determined by the dipole matrix $d_{\ell^{\prime} \ell}=<\chi_{n \ell}|d| \chi_{k \ell^{\prime}}>$ of the dipole operator $d$, either in length or velocity approximation, between the wavefunctions of the initial state $\chi_{n \ell}$ and the final state $\chi_{k \ell^{\prime}}$. Specifically, $f_{f i}$ is given in its length and velocity form, respectively, by

$$
f_{f i}^{L}=\frac{\Delta E_{f i}}{3} d_{\ell^{\prime} \ell}^{2} \quad \text { and } \quad f_{f i}^{V}=\frac{4}{3 \Delta E_{f i}} d_{\ell^{\prime} \ell}^{2}
$$

where $\Delta E_{f i}$ is the excitation energy in $R y$ from the initial state $n \ell$ to the final continuum $k \ell^{\prime}$. Qualitatively, it is clear that the greater the overlap between $\chi_{n \ell}(r)$ and $\chi_{k \ell^{\prime}}(r)$ the larger the cross section. The one-particle wavefunction $\chi$ is calculated by expanding in terms of a set of B-splines defined between $r=0$ to $r=R$. Numerical procedure for $\chi$ is detailed elsewhere $[19,20]$.

For the two-photon ionization, the generalized ionization cross section is given in detailed elsewhere $[19,21,22]$. Within the perturbation theory, the transition amplitude $D^{(2)}$ (see, e.g., Eq. (2) of [22] or Eq. (62) of [19]) of a two-photon ionization from an initial state $n s$ to a final $s(\ell=0)$ or $d(\ell=2)$ electron in the continuum is given by

$$
D^{(2)}=\sum_{\mu} \frac{<\chi_{k \ell}|d| \chi_{\mu p}><\chi_{\mu p}|d| \chi_{n s}>}{\hbar \omega+\epsilon_{n s}-\epsilon_{\mu p}}
$$

where the sum is taken over the complete set of one-electron orbitals of intermediate state $\chi_{\mu p}$ generated by Eq. (4) and $\hbar \omega$ is the energy of the incident photon.

\section{Results and Discussions}

Figure 1 presents the spectra for the single photoionization of hydrogen from its ground state as $D$ decreases from $14.0 a_{o}$ to $12.0 a_{o}$ corresponding to 
the bound-bound $1 s \rightarrow 3 p$ transition and the bound excited $3 p$ state migrates into the continuum due to the less attractive screened Coulomb potential. The $1 s \rightarrow 3 p$ transition appears as a narrow resonance-like structure near the ionization threshold when the $3 p$ state first moves into the continuum and the resonance-like structure gradually de-localizes into broad continuum spectra as $D$ decreases and the $3 p$ state losses its quasi-bound characteristics. This is similar to what was reported earlier for the single photoionization of hydrogen from the excited bound state [9] and the electron-impact ionization from hydrogen atom [13]. This fairly general feature shown in Fig. 1, i.e., the narrow resonance-like structures changing into broad ionization spectra, could be accounted for by a detailed examination of the variation of the wavefunction of the outgoing ionized electron.

Figure 2 shows the quasi-bound characteristics of the $3 p$ state at momenta close to its near resonance values (i.e., they retain most of the bound $3 \mathrm{p}$ characteristics at smaller $r$ region) as $D$ approaches $14.5 a_{o}$ when the bound excited $3 p$ state is pushed into the continuum by the screened Coulomb potential. At momenta $k$ away from the peak of the resonance-like structure, the wavefunctions behave like typical oscillating functions in continuum (see, e.g., the ones corresponding to $k=0.0611111$ and $k=0.0986928$ with $\left.D=13.0 a_{o}\right)$ and the maximum amplitude of the first inner loop of their wavefunctions is noticeably smaller than the one corresponding to the near resonance wavefunction. Since the photoionization cross section is proportional to the square of the dipole matrix $<\chi_{1 s}|d| \chi_{k p}>$ and essentially determined by the overlap of the wavefunctions of the initial bound and the outgoing ionized electrons, only the inner part of the outgoing electron wavefunction up to its first node (i.e., up to a distance $r$ where the wavefunction $\chi_{1 s}$ is still non-negligible) will affect the cross sections. Figure 3 shows the decrease of the local maxima of the inner loop of the quasi-bound wavefunctions as $D$ decreases and, consequentially, a substantial decrease in the square of the dipole matrix element. Since the cross section is also inversely proportional to the momentum $k$, qualitatively, one could then easily conclude that the peak cross section of the photoionization spectra should decrease rapidly as shown in Fig. 1 with decreasing $D$. We should also note that the length and velocity results in all our calculated results agree to four digits or better. Table I presents the peak cross sections of the atomic spectra at varying Debye length $D$ shown in Fig. 1. 
Figure 4 presents the two-photon ionization spectra of hydrogen atom as functions of outgoing photoelectron energy using a linearly polarized light with the Debye lengths $D=\infty$ (i.e., for plasma-free hydrogen atom) and $D=17.5 a_{o}, 14.5 a_{o}$, and $11.5 a_{o}$. Our calculated plasma-free spectrum (with $D=\infty$ ) reproduces exactly the ones published elsewhere [21,22]. As expected, the spectrum is characterized by a series of resonance enhanced $n p$ peaks, due to the energy denominator in Eq. (6), leading to the threshold on the high energy side. The migration of the resonance enhanced $n p$ peaks into the continuum is also clearly shown from the spectra with $D$ changing from $17.5 a_{o}$ to $11.5 a_{o}$ when the $n p$ peaks disappear on the high energy side. In fact, at $D=17.5 a_{o}$, only the $2 p$ and $3 p$ resonance enhanced peaks remain in the calculated spectrum. Whereas on the high energy side, the resonance enhanced $n p$ peaks disappear from the calculated two-photon ionization spectra, the migration of the $3 s$ and $3 d$ states into the continuum have led to the resonance-like structures on the low energy side corresponding to the $2 p \rightarrow 3 d$ and $2 p \rightarrow 3 s$ transitions. It is interesting to note that the presence of the resonance-like structures on the low energy side due to the migration of the $3 s$ and $3 d$ states into the continuum appear even before the resonance enhanced $3 p$ peak disappears completely on the high energy side. This comes from the fact that the contribution to the transition amplitude $D^{(2)}$ from the intermediate $3 p$ state remains substantial due to its quasibound characteristic when it first moves into the continuum.

Figure 5 summarizes the changing of the resonance-like structure into broad ionization spectrum near the threshold at the low energy side corresponding to the $2 p \rightarrow 3 d$ transition as the $3 d$ state migrates into the continuum due to the decrease of the Debye length (or, the increase of the plasma effect). This feature is similar to the de-localization of the narrow resonancelike structure in the single photoionization shown in Fig. 1. Qualitatively, this is due to the variation of the quasi-bound characteristics of the $3 d$ state as it migrates into the continuum. Similar to what was shown in Fig. 2, Fig. 6 presents the one-electron quasi-bound $3 d$ wavefucntions at momenta $k$ close to its "near resonance" values at $D=13.5 a_{o}$ and $15.5 a_{o}$. At momenta $k$ away from the near resonance momentum, the wavefunctions behave more like the oscillating functions in continuum (see, e.g., the ones corresponding to $k=0.097095$ and $k=0.113654$ with $D=13.5 a_{o}$ ). At $D=15.5 a_{o}$ when the bound excited $3 d$ state is barely being pushed into the continuum by the screened Coulomb potential, the wavefunction corresponding to the 
near resonance momentum (i.e., $k=0.0388939$ ) appears almost like a bound $3 d$ state. Figure 7 shows the change of the inner loop of the quasi-bound wavefunctions at their near resonance momenta as $D$ varies from $15.5 a_{o}$ to $13.5 a_{o}$. The substantial decrease in the peak cross sections as $D$ decreases shown in Fig. 5 could be accounted for at least in part by the decrease in the local maxima shown in Fig. 7.

\section{Conclusion}

The variation of the plasma-induced resonance-like atomic structures presented in this paper suggests a fairly general feature of such atomic spectra due to the migration of the upper bound excited states of the bound-bound atomic transitions into the continuum due to the less attractive and shorterranged screened Coulomb potential as the Debye length decreases for an atom embedded in the Debye plasma. The change from sharply narrow resonancelike structure to broad continuous ionization spectrum could be easily and directly accounted for by the overlap between the wavefunctions of the atomic electron in the initial state and its corresponding final ionized state in the continuum.

\section{ACKNOWLEDGMENTS}

This work was supported by the National Science Council in Taiwan under the grant nos. NSC 100-2112-M-030-001, NSC 101-2112M-001-013 and NSC 102-2119-M-007-003.

\section{References}

[1] S. NaKai and K. Mina, Rep. Prog. Phys. 67, 321 (2004).

[2] D. S. Leckrone and J. Sugar, Phys. Scr. T47, 3 (1993).

[3] M. P. Robinson, B. Laburthe Tolra, M. W. Noel, T. F. Gallagher, and P. Pillet, Phys. Rev. Lett. 85, 4466 (2000). 
[4] J. C. Weisheit, Adv. At. Mol. Phys. 25, 101 (1989); G. J. Hatton, N. F. Lane, and J. C. Weisheit, J. Phys. B14, 4879 (1981); B. L. Whitten, N. F. Lane, and J. C. Weisheit, Phys. Rev. A 29, 945, (1984); K. Scheibner, J. C. Weisheit and, N. F. Lane, Phys. Rev. A35, 1252 (1987); X. Lopez, C. Sarasola, and J. M. Ugalde, J. Phys. Chem. A101, 1804 (1997); P. K. Mukherjee, J. Karwowski, and G. H. F. Diercksen, Chem. Phys. Lett. 363, 323 (2002); A. N. Sil and P. K. Mukherjee, Int. J. Quantum. Chem. 102, 1061 (2005); S. Sen, P. Mandal, and P. K. Mukherjee,, Physics of Plasmas 19, 033501 (2012); H. Okutsu, T. Sako, K. Yamanouchi, and G. H. F. Diercksen, J. Phys. B38, 917 (2005).

[5] F. J. Rogers, H. C. Graboske, Jr., and D. J. Harwood, Phys. Rev. A 1, 1577 (1970); U. Gupta and A. K. Rajagopal, Phys. Rept. 87, 259 (1982); N. C. Deb and N. C. Sil, J. Phys. B17, 3587 (1984); F. A. Gutierrez and J. Diaz-Valdes, J. Phys. B27, 593 (1994); R. Brandenburg, J. Schweinzer, S. Fiedler, F. Aumayr, and H. P. Winter, Plasma Phys. Controlled Fusion 41, 471 (1999).

[6] L. B. Zhao and Y. K. Ho, Phys. Plasmas 11, 1695 (2004). Satyabrata Sahoo and Y. K. Ho JQSRT 111, 5262 (2010); Zishi Jiang, Sabyasachi Kar, and Y. K. Ho, Phys. Plasmas 19, 033301 (2012); H. W. Li and Sabyasachi Kar, Phys. Plasmas 19, 073303 (2012); M. Das, Phys. Plasmas 19, 092707 (2012); Sujay Nayek and A. Ghoshal, Phys. Plasmas 19, 113501 (2012); A. Ghoshal, M. Z. M.Kamali, and K. Ratnavelu, Phys. Plasmas 20, 013506 (2013); P. Jiang, S. Kar, and Y. Zhou, Phys. Plasmas 20, 012126 (2013).

[7] R. K. Janev, L. P. Presnyakov, and V. P. Shevelko, Physics of Highly Charged Ions (Springer-Verlag, Berlin, 1985), Chap.3; V. P. Shevelko and L. A. Vainshtein, Atomic Physics for Hot Plasmas, (IOP, London, 1993), Chap. 1.

[8] Z. Wang and P. Winkler, Phys. Rev. A52, 216 (1995); S. T. Dai, A. Solovyova, and P. Winkler, Phys. Rev. E64, 016408 (2001).

[9] C. Y. Lin and Y. K. Ho, Phys. Rev. A81, 033405 (2010); Phys. of Plasmas 17, 093302 (2010); Phys. Scr. T144, 014051 (2011); Comp. Phys. Comm. 182, 125 (2011). 
[10] M. Pandey, Y.-C. Lin, and Y. K. Ho, Phys. of Plasmas 20, 022104 (2013); Phys. of Plasmas 19, 062104 (2012).

[11] S. Paul and Y. K. Ho, Phys. Rev. A78, 042711 (2008); Phys. Rev. A79, 032714 (2009); Phys. Plasmas 16, 063302 (2009); J. Phys. B43, 065701 (2010); Phys. Plasmas 17, 082704 (2010).

[12] S. Kar and Y. K. Ho, J. Quant. Spectrosc. Radiat. Trans. 109, 445 (2008); S. Kar and Y. K. Ho, Phys. Plasmas 15, 013301 (2008); S. Kar and Y. K. Ho, Int. J. Quantum Chem. 106, 814 (2006); A. Ghoshal and Y. K. Ho, J. Phys. B42, 075002 (2009); A. Ghoshal and Y. K. Ho, J. Phys. B42, 175006 (2009).

[13] S. B. Zhang, J. G. Wang, and R. K. Janev, Phys. Rev. A81, 032707 (2010); Phys. Rev. Lett. 104, 023203 (2010); Y. Y. Qi, Y. Wu, J. G. Wang, and Y. Z. Qu, Phys. Plasmas 16, 023502 (2009).

[14] P. Debye and E. Hückel, Phyzik Z. 24, 185 (1923).

[15] H. Margenau and M. Lewis, Rev. Mod. Phys. 31, 569 (1959).

[16] C. A. Rouse, Phys. Rev. 163, 62 (1967).

[17] W. Hong and Y. D. Jung, Phys. Plasmas 3, 2457 (1996); Y. D. Jung, Phys. Fluids B5, 3432 (1993); Y. D. Jung, Phys. Plasmas 2, 332 (1995); Y. D. Jung, Phys. Plasmas 2, 987 (1995); Y. D. Jung, Phys. Plasmas 5, 3781 (1998); Y. D. Jung, Phys. Plasmas 5, 4456 (1998); J. S. Yoon and Y. D. Jung, Phys. Plasmas 3, 3291 (1996).

[18] Y. K. Ho, Phys. Reports 99, 1 (1983).

[19] T. N. Chang, in Many-body Theory of Atomic Structure and Photoionization, edited by T. N. Chang (World Scientific, Singapore, 1993), p. 213.

[20] T. N. Chang and T. K. Fang, Radiat. Phys. Chem. 70, 173 (2004); T. N. Chang and T. K. Fang, Phys. Rev. A52, 2638 (1995); T. N. Chang and X. Tang, Phys. Rev. A44, 232 (1991); T. N. Chang, Phys. Rev. A39, 4946 (1989).

[21] T. N. Chang and R. T. Poe, J. Phys. B9, L311 (1976); T. N. Chang and R. T. Poe, Phys. Rev. A16, 606 (1977). 
[22] X. Tang, T. N. Chang, P. Lambropoulos, S. Fournier, and L. F. DiMauro, Phys. Rev. A41, 5265 (1990).

Table I. The peak cross sections of the atomic spectra at varying Debye length $D$ shown in Fig. 1.

\begin{tabular}{clr}
\hline \hline $\mathrm{D}\left(\right.$ in $\left.a_{o}\right)$ & $k^{2}($ in $\mathrm{eV})$ & $\sigma_{\text {peak }}($ in $\mathrm{Mb})$ \\
\hline \hline & & \\
14.0 & 0.0296 & 496 \\
13.5 & 0.0562 & 170 \\
13.0 & 0.0840 & 79.0 \\
12.5 & 0.113 & 43.5 \\
12.0 & 0.148 & 28.2 \\
& & \\
\hline \hline
\end{tabular}




\section{Figure captions}

Figure 1: Plasma-induced resonance-like photoionization spectra of hydrogen atom corresopnding to the $1 s \rightarrow 3 p$ transition as $D$ varies.

Figure 2: The radial wavefunction $\chi_{k p}$ of the outgoing ionized electron of hydrogen with $D=13 a_{o}$ and $14 a_{o}$ at a number of momenta.

Figure 3: The comparison of quasi-bound radial wavefunction $\chi_{k p}$ of the outgoing ionized electron of hydrogen at a number of $D$ values.

Figure 4: Two-photon ionization spectra of hydrogen with outgoing ionized $s$ and $d$ electrons as $D$ varies.

Figure 5: Plasma-induced resonance-like two-photon ionization spectra of hydrogen atom near the ionization threshold corresponding to the $2 p \rightarrow 3 d$ transition as $D$ varies.

Figure 6: The radial wavefunction $\chi_{k d}$ of the outgoing ionized electron of hydrogen with $D=13.5 a_{o}$ and $15.5 a_{o}$ at a number of momenta.

Figure 7: The comparison of quasi-bound radial wavefunction $\chi_{k d}$ of the outgoing ionized electron of hydrogen at a number of $D$ values. 


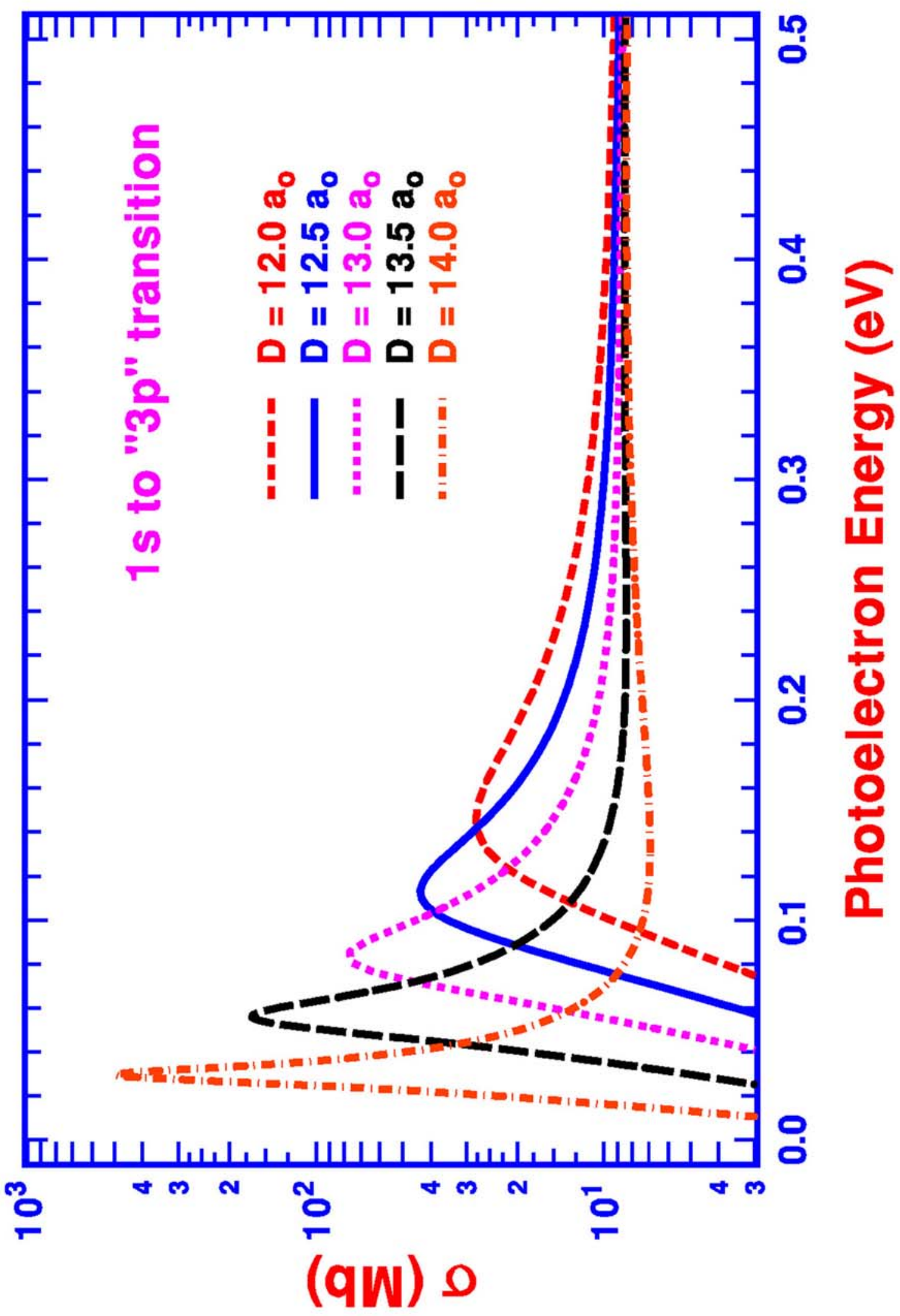



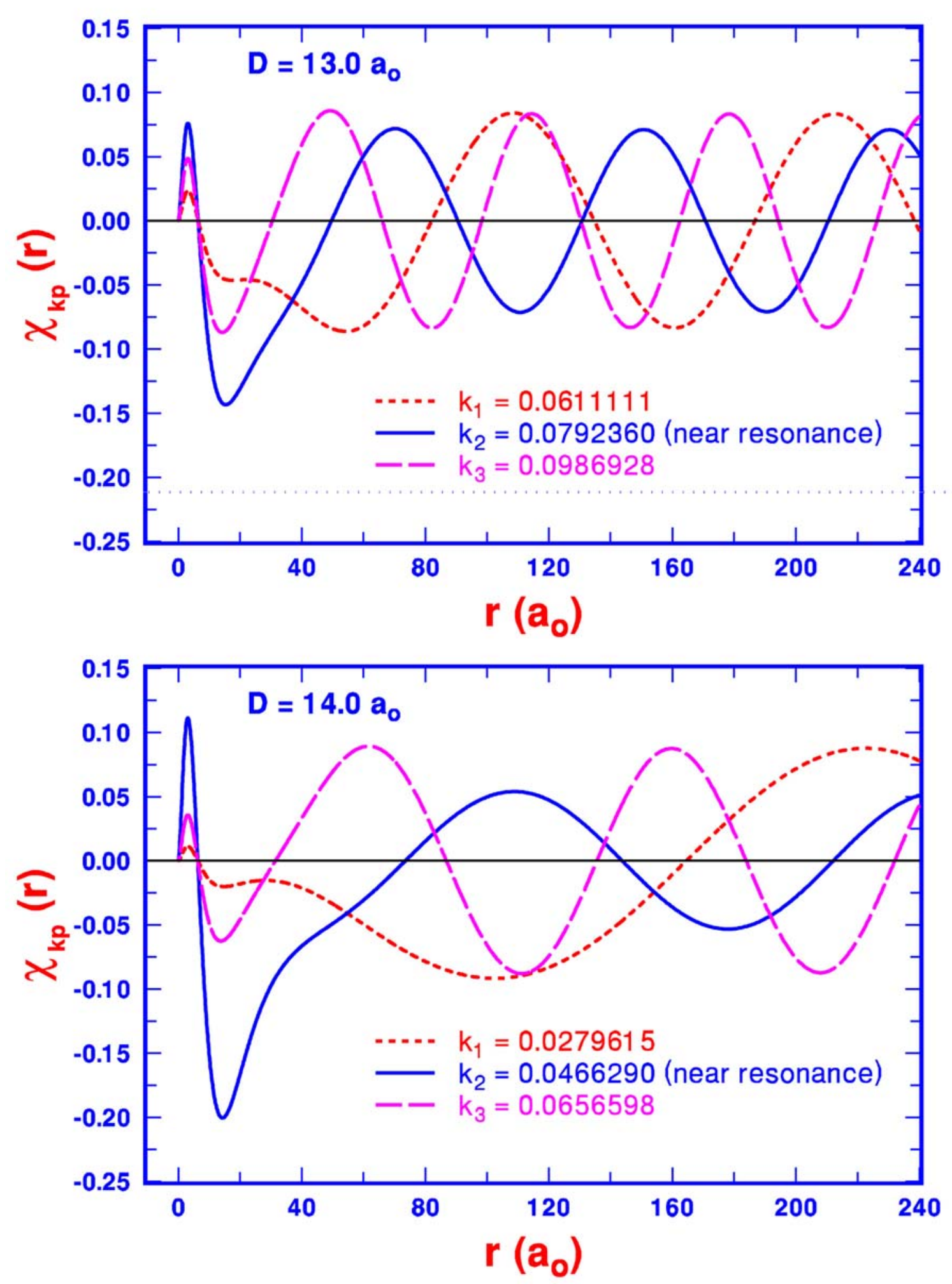


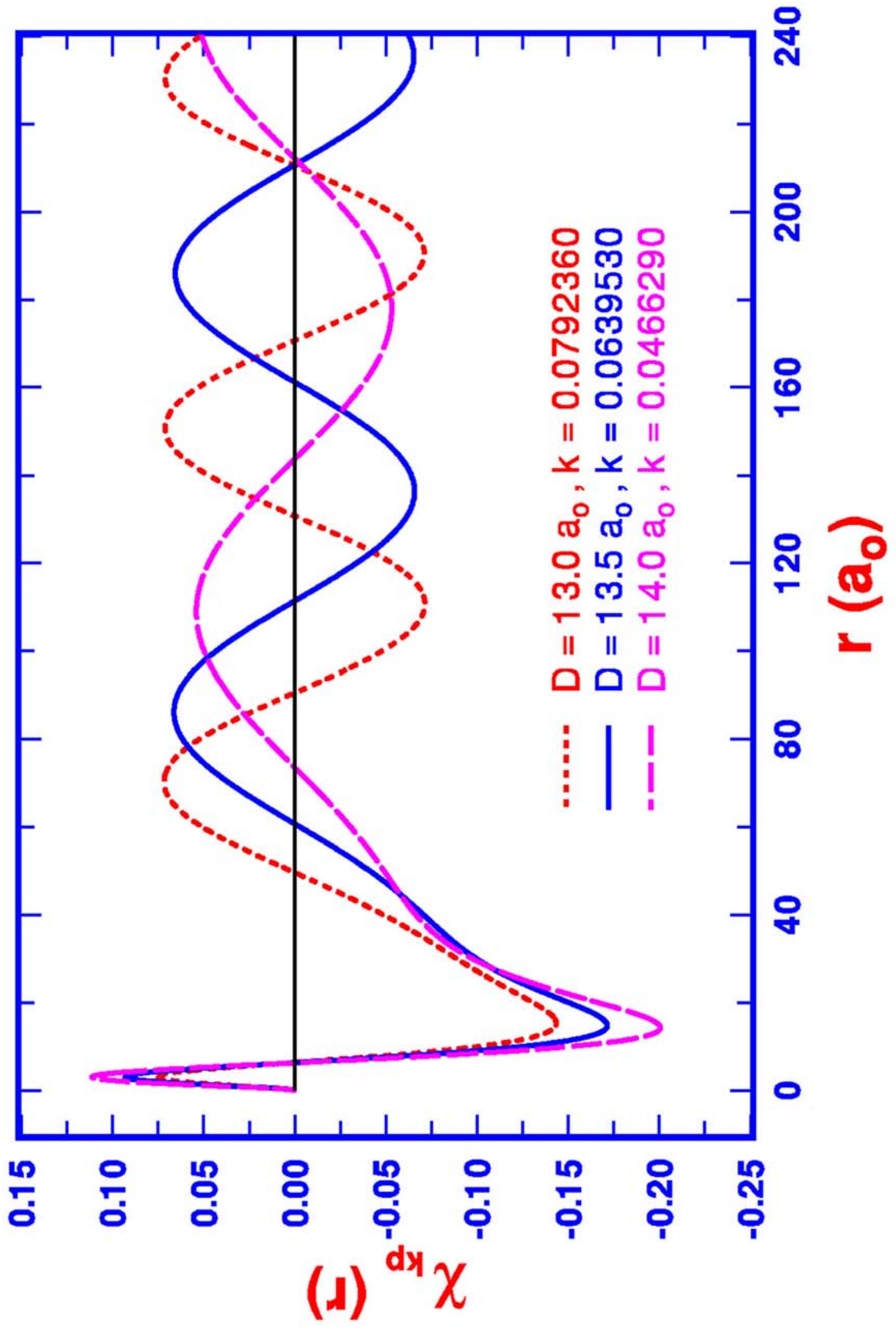



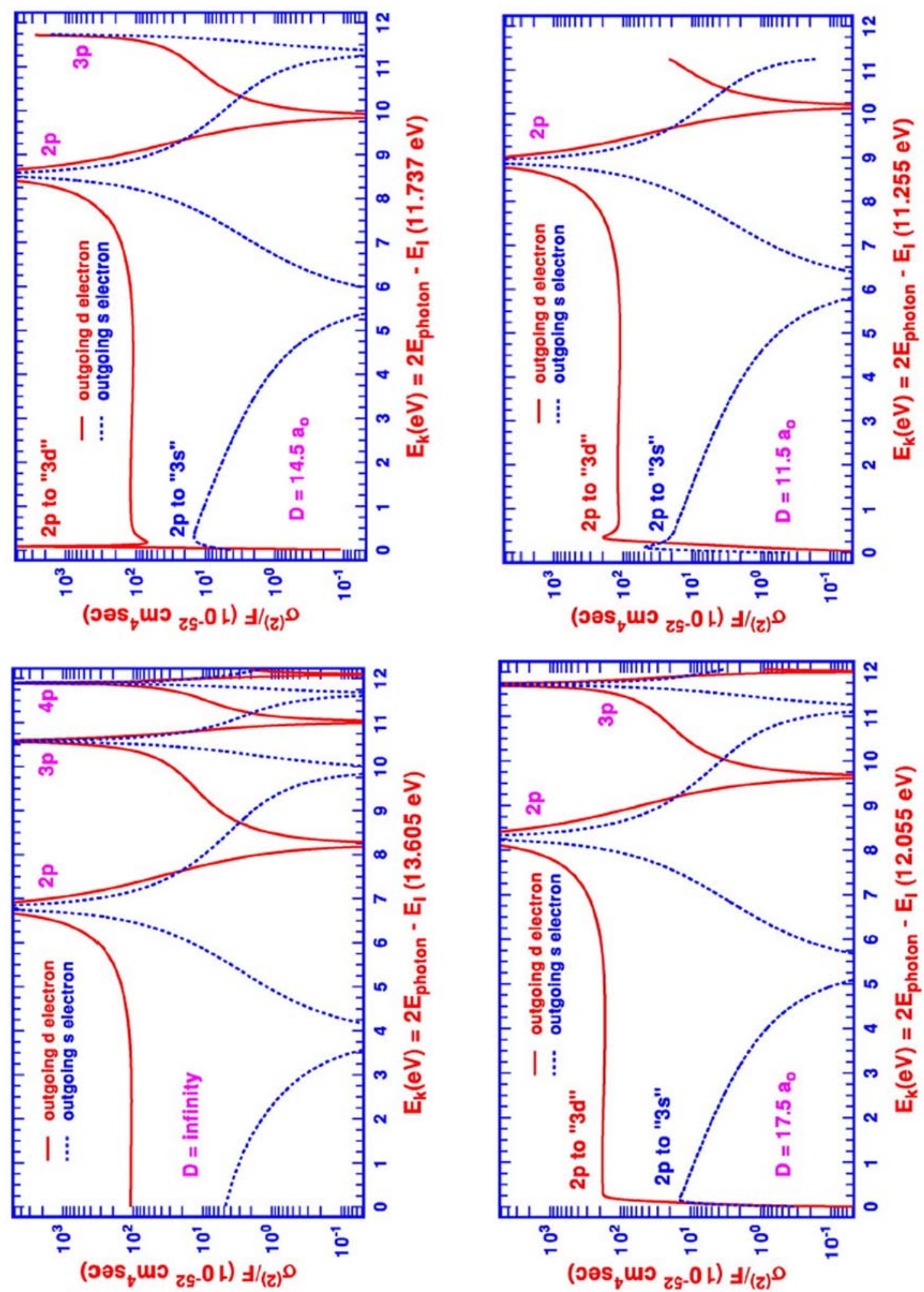


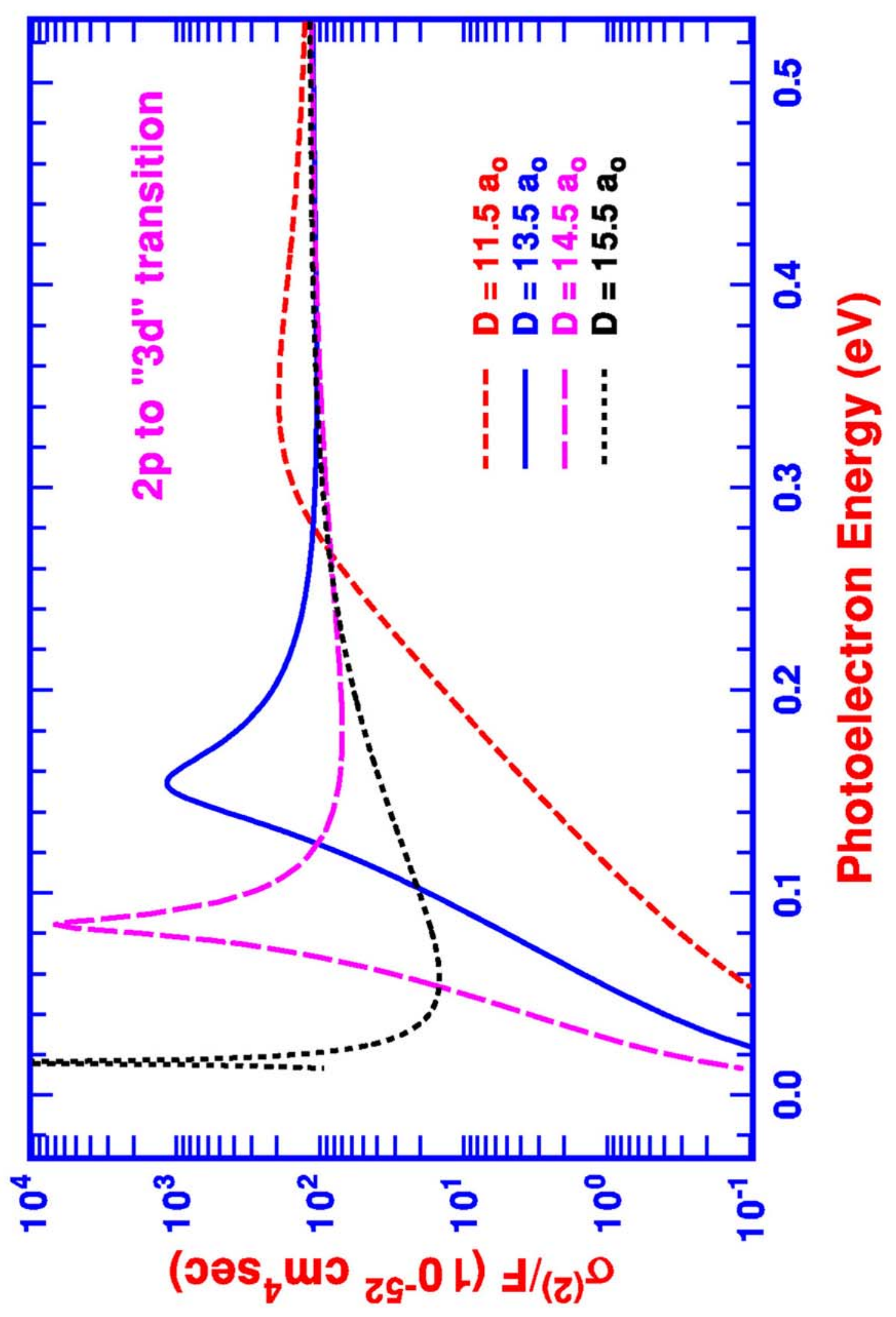



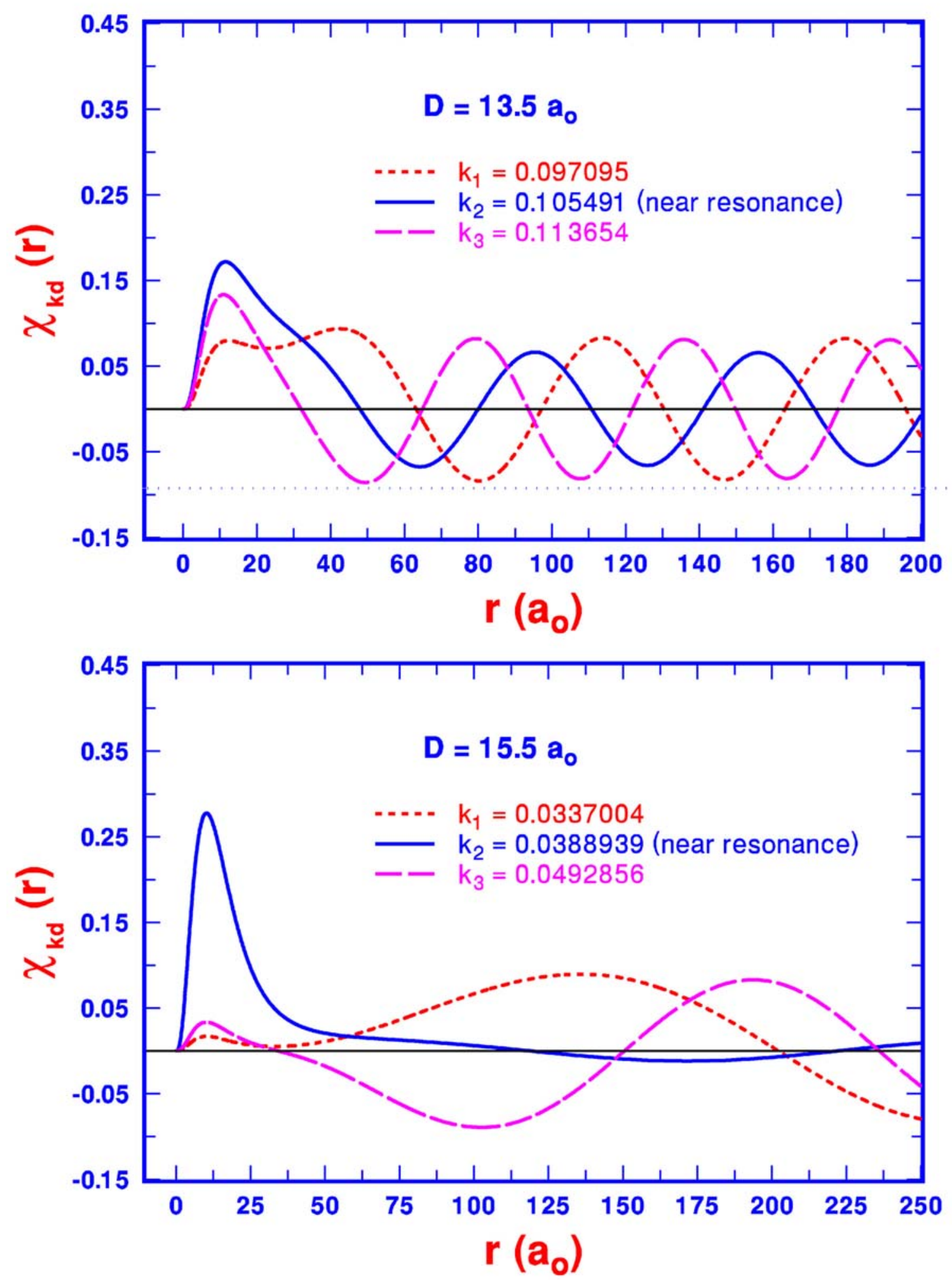


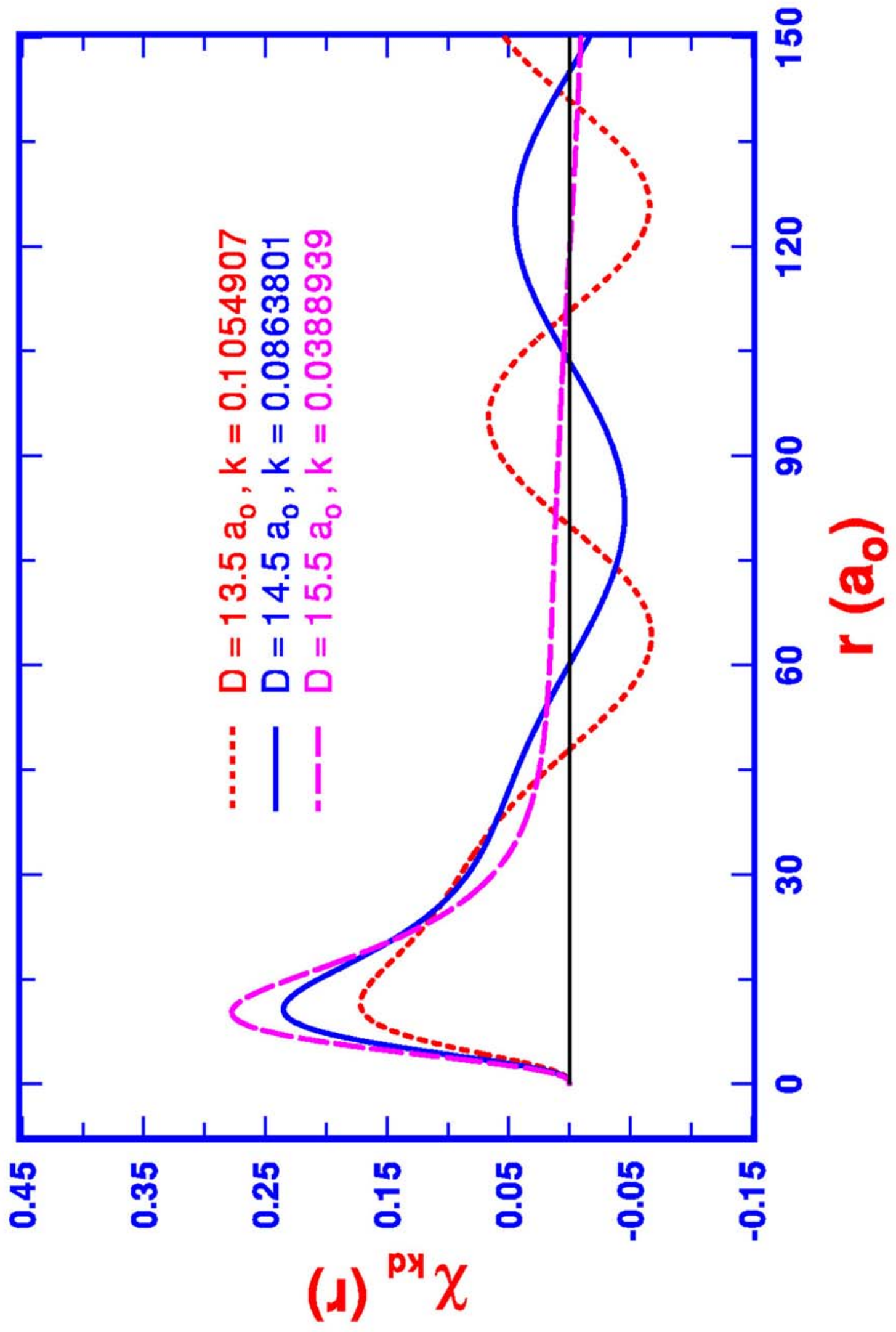

